\title{
The design and analysis of flip structure of the automatically flip packing container for spacecraft
}

\author{
ZHU Ya-hong ${ }^{1, a}$, YANG Chun-sheng ${ }^{1}$ and MENG Shao-hua ${ }^{1}$ \\ NO.104, Youyi Road, Haidian, Beijing. PO CONTAINER 5142-88 100094 China
}

\begin{abstract}
The automatically flip container is specially designed in order to solve the problem $t$ of limited lifting height.Theflip container cover is realized by using the electric push rod mechanism. Compared with the traditional packing container, this container can be opened without lifting the container cover. Due to the automatically flip structure, it is easy to open the container under the condition of limited fixed facilities.
\end{abstract}

\section{INSTRUCTION}

The packing container makes role of important equipment for spacecraft transportation ${ }^{[2]}$, which can guarantee the storage environment ${ }^{[3]}$ for the spacecraft. The traditional type of spacecraft packing container is a split type structure, which consists of the bottom and the cover.The crane hoisting must be used when hoisting container cover out of the container, therefore, the lifting and carrying capacity of the crane is required to have the corresponding capacity. However, with the development of spacecraft and the limitation of fixed facilities larger and larger, if we can't meet the requirements of open container lifting, we need to develop a new packaging container which can open the container under the condition of limited fixed facilities to meet the needs. This paper introduces the flipping structure design of a flipping type packing container. The joint part is the hinge structure which locates between bottom and cover. The electric push rod is used to provide the power driving to make cover rotate along the hinges to realize flipping of container.

\section{Flipping Structure design}

\subsection{Overall design}

In the design scheme, The outsize of the spacecraft required to be transported is $2800 \mathrm{~mm} \times 2500 \mathrm{~mm} \times 2900$ mm(length $\times$ width $\times$ height), the closing state of the packing container is shown in Figure 1, and the opening state of the packing container is shown in Figure 2.The biggest difference between the packing container and the common turnover device is that after closing the cover, it can ensure the container has good sealing property. In this design, the inflatable sealing ring is used to meet the sealing property requirement of the container.
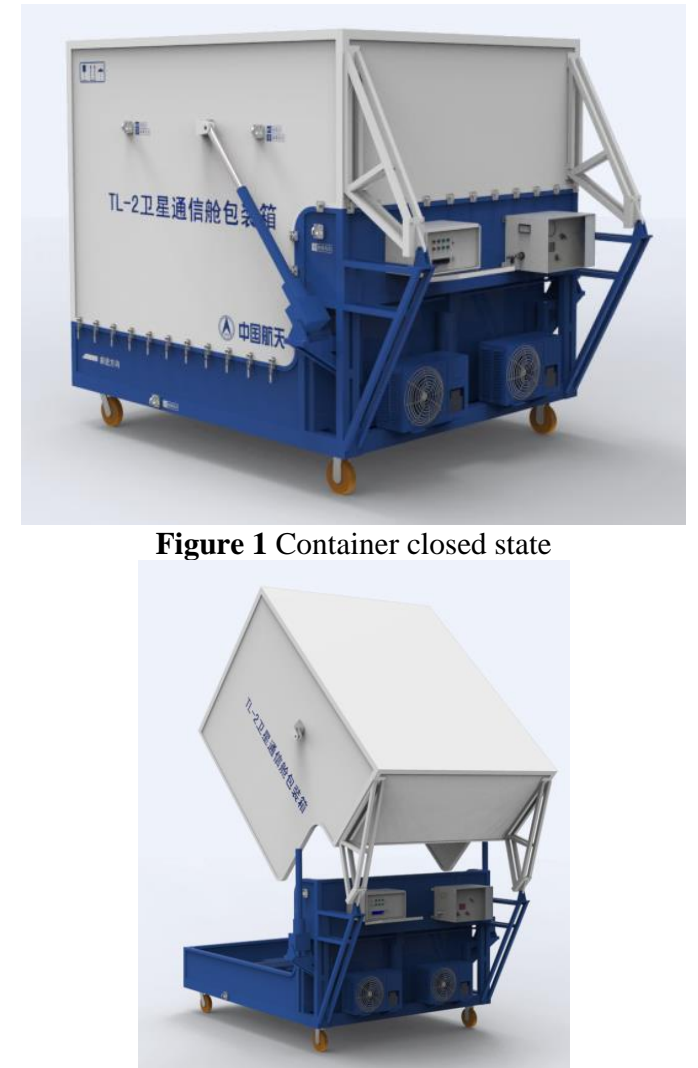

Figure 2 Container opened state

\subsection{Flipping structure}

Flipping structure is mainly composed of a container cover and a container bottom and electric push rod. The joint is the hinge structure bottom and a container cover, In order to realize the flipping action, the hinge connection point is designed in a high position, and the electric push rod is connected to the cover, which provides power for driving the cover to turn over. The push rod is the key part of the turnover mechanism. The position of the push rod, the required stroke and the 
thrust affects the original length of the push rod together. In this scheme, the container cover is about $1200 \mathrm{~kg}$, and the two push rods are synchronized, and the spacecraft can be moved out of the packing container after the cover is turned over $85^{\circ}$.The selection of the original length of the push rod $2055 \mathrm{~mm}$, travel $1536 \mathrm{~mm}$, thrust 5T, which pushes the container to the maximum angleas shown in Figure 3.

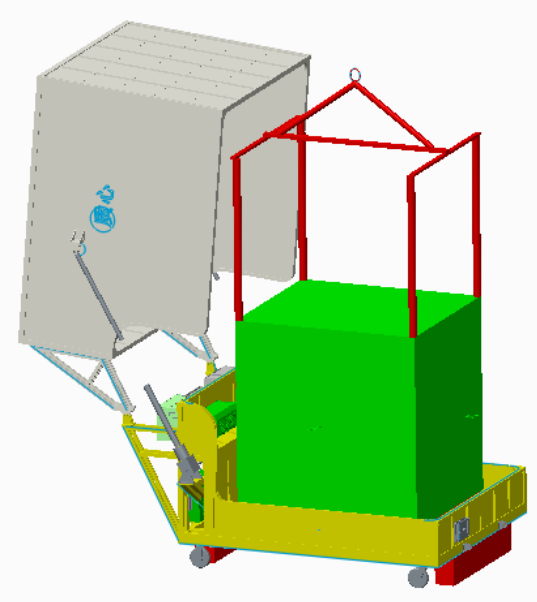

Figure 3 Turn to the $85^{\circ}$

\section{Mechanical analysis}

\subsection{Mechanics principle}

The mechanical coordinate system of the system is shown in Figure 4. The movement in the whole process is very slow, which can be seen as a static problem. The mechanical model is concentrated load made up of a turning body (container cover), which is used to turn the back fulcrum into a rotating shaft as a rotating motion system. In the course of the movement, the external force of the container is described as follows: center of gravity $\mathrm{W}$; fulcrum of the force can be decomposed into vertical $\mathrm{N}$ and horizontal component $\mathrm{T}$; axial force in the direction along the front fulcrum of the electric push rod $\mathrm{F}$, because the container cover revolves around the pivot point $\mathrm{O}$, the gravity $\mathrm{W}$ and $\mathrm{F}$ do not pass through the $\mathrm{O}$ point, so a set of relative moment interval $s$ of the $O$ point are generated. Taking $\mathrm{O}$ as the original point, the coordinate system of $\mathrm{O}-\mathrm{X}$ and $\mathrm{Y}$ is set up, and the static mechanical equations are set up. The value and direction of each force can be obtained. The connection dimensions are shown in Figure 5.

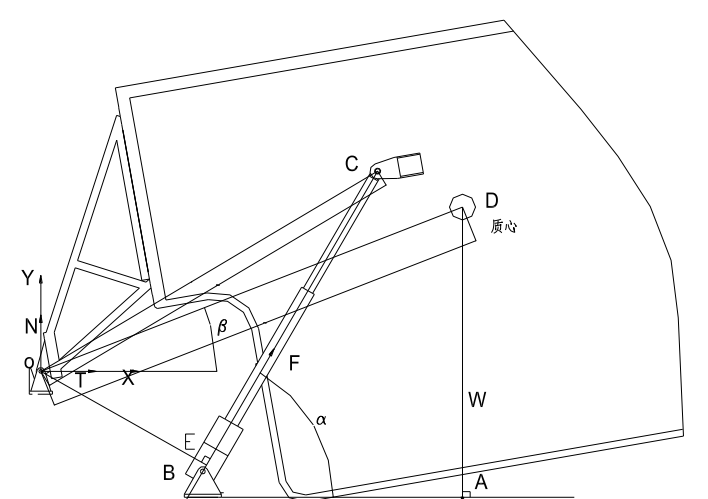

Figure 4 Relationship between mechanical coordinate system

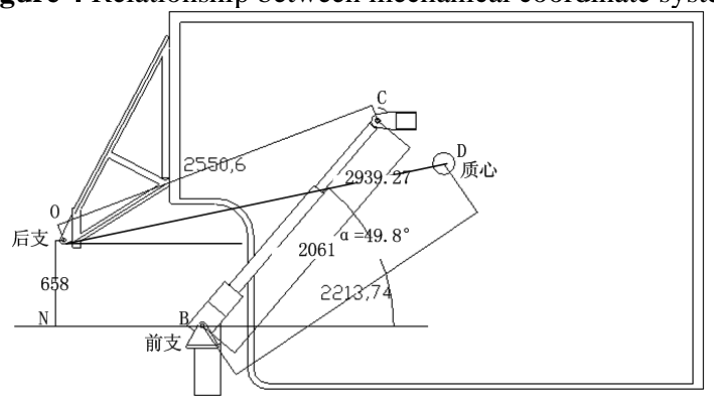

Figure 5 Flip structure connection relationship dimension

The statics equation is shown as follows(1)、(2)、(3);

$$
\begin{array}{ll}
\sum \mathrm{M}=0 & \mathrm{~W} \cdot \mathrm{OA}=\mathrm{F} \cdot \mathrm{OE} \\
\sum \mathrm{F}_{\mathrm{X}}=0 & \mathrm{~T}+\mathrm{F} \operatorname{Cos} \alpha=0 \\
\sum \mathrm{F}_{\mathrm{Y}}=0 & \mathrm{~N}+\mathrm{F} \operatorname{Sin} \alpha=\mathrm{W}
\end{array}
$$

The detailed data are as follows:

$\mathrm{W}=1200 \mathrm{~kg}$;

$\mathrm{ON}=658 \mathrm{~mm}$;

$\mathrm{OD}=2939.27 \mathrm{~mm}$;

$\mathrm{OC}=2550.6 \mathrm{~mm}$;

Set the $\beta$ as the known angle, it starts from $0^{\circ}$ with the interval is $10^{\circ}$. Through the software simulation, we can measured a and calculate $F$. Take the a and $F$ values into (2) and (3), then we can get the $\mathrm{N}$ value and the $\mathrm{T}$ value, so the result list is shown in table 1 .

Table 1. Result of analysis

\begin{tabular}{crrrrr}
\hline \multicolumn{1}{r}{ 力 } & $\beta$ & a & $\mathrm{F}$ & $\mathrm{T}$ & \multicolumn{1}{c}{ Unit: KN } \\
\hline 1 & $0^{\circ}$ & $49.86^{\circ}$ & -28.17 & 18.16 & 9.54 \\
2 & $10^{\circ}$ & $60.17^{\circ}$ & -26.49 & 13.17 & 10.98 \\
3 & $20^{\circ}$ & $69.52^{\circ}$ & -24.78 & 8.6 & 11.21 \\
4 & $30^{\circ}$ & $78.21^{\circ}$ & -22.71 & 4.52 & 10.23 \\
5 & $40^{\circ}$ & $86.38^{\circ}$ & -20.14 & 1.27 & 8.09 \\
6 & $50^{\circ}$ & $94.16^{\circ}$ & -16.8 & -1.21 & 4.76 \\
7 & $60^{\circ}$ & $101.66^{\circ}$ & -12.46 & -2.51 & 0.2 \\
8 & $70^{\circ}$ & $108.9^{\circ}$ & -6.64 & -2.15 & -5.7 \\
9 & $80^{\circ}$ & $115.98^{\circ}$ & 1.44 & 0.63 & -13.3 \\
10 & $85^{\circ}$ & $119.47^{\circ}$ & 6.79 & 3.34 & 17.9 \\
\hline
\end{tabular}

The results of the list are as follows: When $a=0^{\circ}$, the electric push rod has the largest $\mathrm{F}$ force, the largest $\mathrm{T}$ force, when $\mathrm{a}=85^{\circ}$, the electric push rod has the largest $\mathrm{N}$ force. The data result is the basis for checking the strength and stiffness of the component. 


\subsection{Mechanical checking}

The materials of container cover and bottom are 5A06. The $\sigma_{0.2}=145 \mathrm{Mpa}$, Poisson's ratio $=0.33$, elastic modulus $=69 \mathrm{GPa}$, as input for finite element analysis of the structure.

The mechanical analysis of the connection point of the container cover and the electric push rod is described as follows. Single point loading $14085 \mathrm{~N}$ $(28.17 / 2=14.085 \mathrm{KN})$, the results of mechanical analysis are shown in figures 6 and 7 . The maximum stress is $22.6 \mathrm{MPa}$ which meets the 3 times safety factor ${ }^{[4]}$, Maximum displacement can reach $1.2 \mathrm{~mm}$, the deformation has no effect on the opening and closing of the container. So it can meet the requirements of stiffness $^{[5]}$.

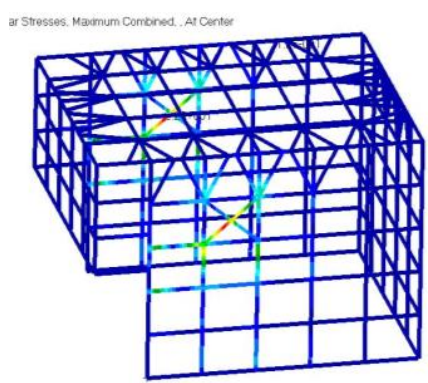

Figure 6 The container cover stress analysis

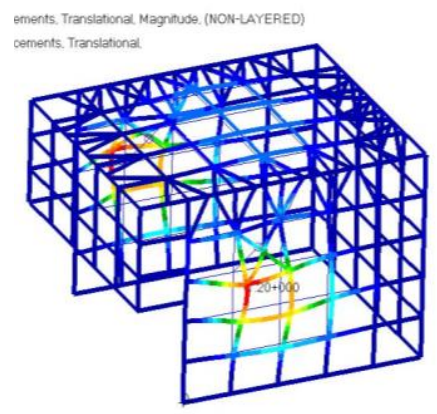

Figure 7 The container cover deformation analysis

The mechanical analysis of the container cover support frame and bottom connection, loading $\mathrm{T}=18000 \mathrm{~N}, \mathrm{~N}=9500 \mathrm{~N}$, the results of mechanical analysis are shown in figures 8 and 9.The maximum stress reach $23.8 \mathrm{MPa}$, meet the 3 times safety factor. Maximum displacement reach $0.98 \mathrm{~mm}$, the deformation has no effect on the opening and closing of the container So it can also meet the requirements of stiffness.
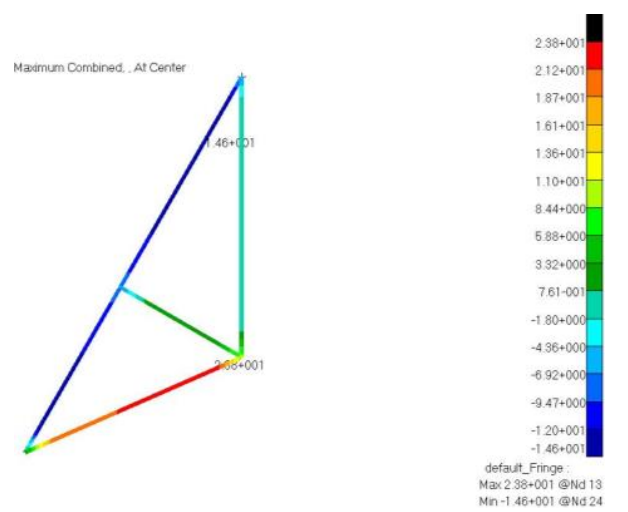

Figure 8 The container cover support stress analysis
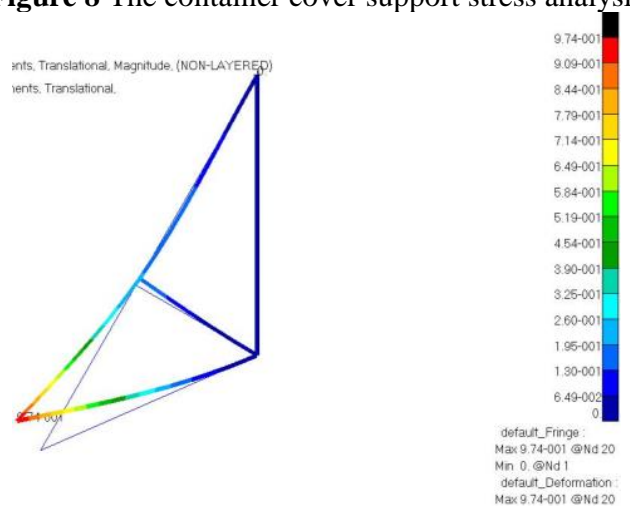

Figure 9 The container cover support deformation analysis

The mechanical analysis of the connection point of the container bottom and the electric push rod is described as follows. The resul of mechanical analysis are shown in figures 10 and 11 . The maximum stress values $50 \mathrm{MPa}$ meeting the 3 times safety factor. Maximum displacement reach $0.1 \mathrm{~mm}$, the deformation has no effect on the opening and closing of the container. So it can meet the requirements of stiffness.
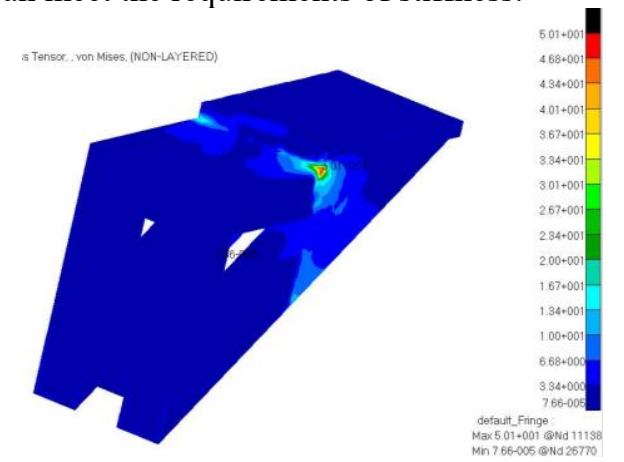

Figure 10 The container bottom stress analysis
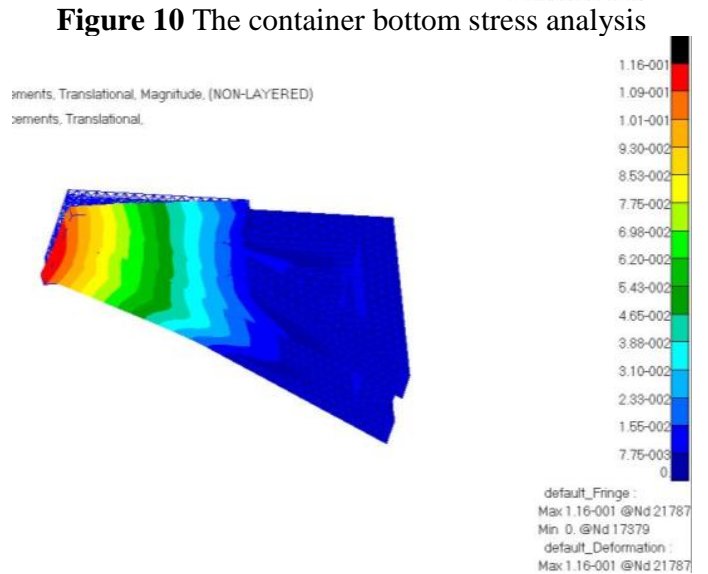

Figure 11 The container bottom deformation analysis 
The mechanical analysis of the container bottom support frame and cover connection , loading $\mathrm{T}=3300 \mathrm{~N}, \mathrm{~N}=18000 \mathrm{~N}$, the results of mechanical analysis are shown in figures 12 and 13.The maximum stress values $50 \mathrm{MPa}$ meeting the 3 times safety factor. Maximum displacement can reach $0.1 \mathrm{~mm}$. The deformation has no effect on the opening and closing of the container. So it can also meet the requirements of stiffness.
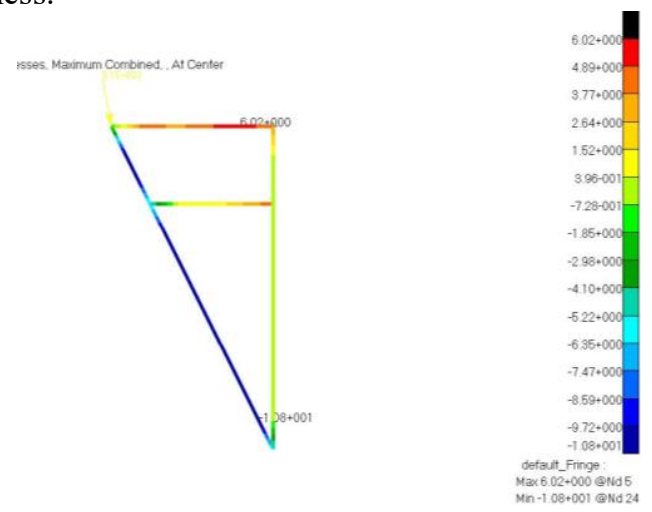

Figure 12 The container bottom support stress analysis

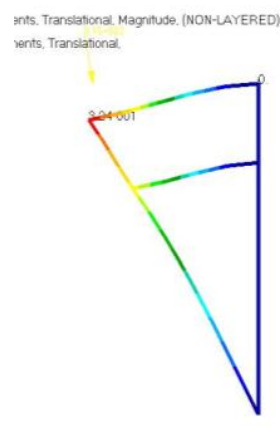

Figure 13 The container bottom support deformation analysis

\section{Concluding remarks}

This paper introduces the design of the flip structure of the flipping container. During the flipping process, with changing of the opening angle guadually, the mechanical environment of the container will change. According to the change of mechanics, the mechanical analysis of the structure of the container is carried out and checked. This type of structure design can be used as a reference.

\section{References}

1. Hu. Qizheng, Yang. Fang, Introduction to Astronautics, P376 379 (2010)

2. G. Xiao, F.Guo, F Cong,The development of containers for air transport of spacecraft and an evaluation [J]. SEE, 27(6):P795-796.(2010)

3. LM Chen,Spacecraft structure and mechanism, P80 81 (2005)

4. GB/T 32301-2015, General requirements of packaging and transportation for spacecraft
5. ISO14625, space systems-Ground support equipment for use at launch, landing or retrieval sites-General requirement 